\title{
Charging Effects in Self-Assembled CdTe Quantum Dots
}

\author{
Ł. KŁOPOTOWSKI*
}

Institute of Physics, Polish Academy of Sciences, al. Lotników 32/46, 02-668 Warsaw, Poland

\begin{abstract}
In this review, we summarize our achievements in controlling and understanding the charging effects in single self-assembled CdTe quantum dots. We start with analysis of the single dot emission spectrum. For excitation densities small enough to ensure only $s$-shell recombination, we find that for all dots (also those reported by other groups) the same transition sequence is observed. Namely, the neutral exciton recombination has the highest energy while charged exciton and biexciton transitions are redshifted. This observation remains in a stark contrast to self-assembled InGaAs dots, where charged complexes may appear also on the high energy side of the neutral exciton. We explain the universality of the transition sequence assuming domination of the Coulomb correlations over direct, single particle interactions. Furthermore, through measurement of the recombination rates, we gain access to electron and hole wave functions and their redistributions upon changing the dot occupancy. We find that the electron wave function is rather stiff, while the hole wave function is rather soft owing to enhanced correlations in the valence band. We then corroborate these conclusions with the Stark spectroscopy, where we analyze energy shifts due to electric field imposed on dots embedded in a field effect or diode structure. Finally, we use these structures to obtain controllable tuning of the charge state. We discuss different approaches to this task and find the best tuning efficiency for a structure with enhanced valence band confinement.
\end{abstract}

PACS: 78.67.Hc, 32.60.+i, 71.35.Pq, 71.55.Gs

\section{Introduction}

Three-dimensional confinement of carrier motion in semiconductor quantum dots (QDs) make them ideal candidates for many applications in novel electronic and optoelectronic devices. In particular, due to relatively weak coupling of zero-dimensional carriers with the environment, the dots are proposed as building blocks of future quantum information processing devices [1], relying on qubits robust against decoherence. Moreover, because of the zero-dimensional density of states, QD are often referred to as artificial atoms [2] and indeed many physical phenomena previously known only from atomic physics have been observed in QDs. These include effects related to strong light-matter coupling [3], observation of the Rabi oscillations [4-6], emission of non-classical light [7], and emission of entangled photons $[8,9]$.

However, unlike atoms, self-assembled QDs can be easily embedded into other semiconductor devices. It allows not only to study novel effects related to zero-dimensional structures, but also to exploit the atomic-like energy structure of QDs in devices. One of the most important possibilities is to embed the dots in a field effect [10] or diode [11] structure and, by changing a bias, tune the QD charge state [12]. Sequential injection of electrons into the dots is possible because of a Coulomb blockade: addition of an electron raises the electrostatic barrier for adding another one - another feature of the

* e-mail: lukasz.klopotowski@ifpan.edu.pl zero-dimensional density of states [13]. By tuning the bias, controlling the charge state of a single dot in the range between $-8 e$ and $+6 e$ was demonstrated [14] and allowed to assess the way the QD occupancy builds up. By measuring the photoluminescence (PL) spectrum, it was shown that the electrons fill the QD shells according to the Hund rule and the Aufbau principle, but for the holes both of these rules are violated [15, 16]. Application of a bias in the field-effect structure result in an electric field and thus makes possible the studies of a quantum confined Stark effect (QCSE) [17, 18]. The magnitudes of the Stark shifts of the excitonic transitions are sensitive to the charge distributions within a dot [19] and therefore provide a spectroscopic access to QD morphology and charge redistributions upon changing the dot occupancy [20]. On the other hand, electric field enables separation of the photocreated electron-hole pair by tunneling one of the carriers ahead of the other. The resulting photocurrent can be used as a spectrum analyzer with ultrahigh bias-tunable sensitivity [21]. In particular, it can provide a tool for studies of single electron coherence $[21,22]$ and two-qubit conditional logic operations $[23,24]$. On the other hand, the isolated carrier can be exploited as a single spin memory device [25, 26]. Moreover, separating the electron in a dot enabled a direct measurement of its transverse spin relaxation time [25].

Most of the QD research concentrates on the InGaAs dots, where growth procedures are well established. Indeed, coherent control of a QD exciton - an important prerequisite for quantum information applications - was demonstrated as early as in 1998 [4]. The main obstacle 
on the way to implementation in quantum devices is decoherence of the qubit. Its major source was identified as resulting from interaction with the nuclei of the atoms building a dot $[27,28]$, which provide a magnetic-like fluctuating field. Control of the dot charge state made it possible to monitor and control the polarization of the nuclei [29-31]. CdTe dots offer an interesting alternative with an advantage of smaller hyperfine interactions. Natural abundances of isotopes with a non-vanishing spin is four times smaller in CdTe compared to InAs, and nuclear magnetic moments are 3 to 9 times smaller. Controlling the charge state should allow to evaluate the strength of the hyperfine interactions in a CdTe dot. Another important advantage of CdTe dots over those of InGaAs is the possibility of isoelectronic doping with multiple Mn ions $[32,33]$. Electrical control of the charge state of such semimagnetic dots is expected to enable tailoring and switching the magnetization on and off [34-36]. Hence, in order to exploit the advantages of CdTe as the QD material, controllable charging is crucial.

In this report, we summarize our achievements in control and understanding of charging effects in single CdTe QDs. We start with a short description of CdTe QD fabrication methods and present basic spectroscopic properties of these dots. We then discuss the shape of the emission spectrum under low excitation density, where only the lowest electron and hole shell is occupied. We focus on the universality of the transition sequence and relate it to strong Coulomb correlations. We compare the spectroscopic signatures to those characteristic of self-assembled InAs dots and GaAs dots formed on monolayer well width fluctuations ("natural" dots). In the next section, we investigate further the Coulomb correlations by analyzing the decay rates of the PL transitions. We find that the confinement conditions in these dots are rather far from the strong confinement limit and that the recombination rates depend on the dot occupancy. In the subsequent section, we study charging behaviors of three different structures designed to provide charge tunability. In all of these structures, the dots are embedded in a depletion region of a diode structure. The electric field resulting from the applied bias gives rise to the QCSE. We analyze it in the usual framework of perturbation theory and extract information on charge distributions and their modifications in electric field. In the last section, we conclude and provide a (somewhat subjective) outlook on the studies of these CdTe dots.

\section{Fabrication and basic properties of CdTe quantum dots}

CdTe dots grown by molecular beam epitaxy (MBE) are usually formed via a modified Stranski-Krastanov procedure. In the method currently applied most often, a strained layer of CdTe (width not exceeding the critical thickness for dislocation formation) is deposited at $\approx 280^{\circ} \mathrm{C}$. The growth temperature is then lowered to room temperature and the layer is covered with amorphous tellurium [37] or zinc [38]. After increasing the temperature to $\approx 220^{\circ} \mathrm{C}$, the amorphous layer is desorbed and the dots are formed as a result of changing the balance between the elastic and surface energies. The dots are approximately lens-shaped, with base diameter in the range between 20 and $40 \mathrm{~nm}$, and heights from 2 to $8 \mathrm{~nm}$ [37] and their density is on the order of $10^{10} \mathrm{~cm}^{-2}$. Another fabrication method involves a deposition of the strained CdTe layer at an elevated temperature of $\approx 420^{\circ} \mathrm{C}$, whereupon the dots form spontaneously. In this case the dots are substantially smaller, with diameters of about 2-4 nm [39, 40]. The QD density is as high as $10^{12} \mathrm{~cm}^{-2}$ [41], which makes single dot studies more difficult. The former growth mechanism allows to obtain vertically stacked dots, as in the case of InAs system [42], whereas in the latter, QD layers rather form a superlattice, in which the dots in subsequent layers spatially avoid each other [43].

Zero-dimensional density of states of these structures is confirmed by e.g., measurement of the PL lineshape as a function of temperature. At $5 \mathrm{~K}$, the PL transition has a shape of a Lorentzian with a linewidth of $\approx 150 \mu \mathrm{eV}$. This zero-phonon line broadens as the temperature is increased and simultaneously phonon side-bands develop [44]. Single photon emission was demonstrated by photon correlation measurements in a Hanbury-Brown and Twiss setup [45], which revealed antibunching of photons coming from the same transitions and bunching in a biexciton-exciton cascade [46]. Unfortunately, these dots exhibit anisotropic exchange splitting of the neutral exciton ground state [47] analogous to the one observed in the InGaAs QDs [48], hindering the emission of entangled photons. In the CdTe case however, the direction of the anisotropy varies from dot to dot and is not related to any crystallographic axes [47]. The energy levels of these dots exhibit an atomic-like shell structure as demonstrated by high excitation spectroscopy [49].

As mentioned in Introduction, CdTe dots can be easily doped with $\mathrm{Mn}$ ions. In particular, a dot with a single $\mathrm{Mn}$ ion can be fabricated $[50,51]$. It allows to manipulate and read out the state of a single ion with spectroscopic tools. In particular, optical orientation of the Mn spin was demonstrated by selective excitation either resonantly [52] or quasi-resonantly via a tunnel-coupled CdTe dot [53]. Importantly, charge control of a dot with a single Mn ion was demonstrated. It provided a direct method of tailoring the spin properties of the exciton-Mn ion system [54].

PL excitation experiments on CdTe dots reveal quasi two-dimensional excited states [40], which replace the wetting layer usually present in InGaAs system. It is proposed that these states originate from micrometer sized platelets, on which the dots are grown [55]. Also, a quasiresonant excitation via LO phonon-assisted process was demonstrated [40], however it appears that it is only efficient for dots with diameters below $10 \mathrm{~nm}$. Single dots spectroscopy reveals transitions related to different occupancies of a dot. Upon non-resonant continuous 
excitation, transitions related to different charges states coexist [56] due to a non-geminate carrier capture occurring on a sub-nanosecond time scale [57]. In the following section, we discuss the details of the single dot emission spectrum.

\section{Single dot emission spectrum}

In Fig. 1, we show PL spectra for two different dots grown from 2 and 6 ML thick CdTe layers using the tellurium desorption procedure [37] described above. The excitation wavelength is $532 \mathrm{~nm}$, providing below barrier pumping. In both cases, the spectra consist of four major transitions with the one at highest energy separated from the remaining three. We identify these transitions as recombinations of a neutral exciton $\left(\mathrm{X}^{0}\right)$, two charged excitons $\left(\mathrm{X}^{+}\right.$and $\left.\mathrm{X}^{-}\right)$, and a biexciton $(\mathrm{XX})$ in a sequence where $E_{\mathrm{X}^{0}}>E_{\mathrm{X}^{+}}>E_{\mathrm{X}^{-}}>E_{2 \mathrm{X}}$, where $E_{\chi}$ is the emission energy of the complex $\chi$.

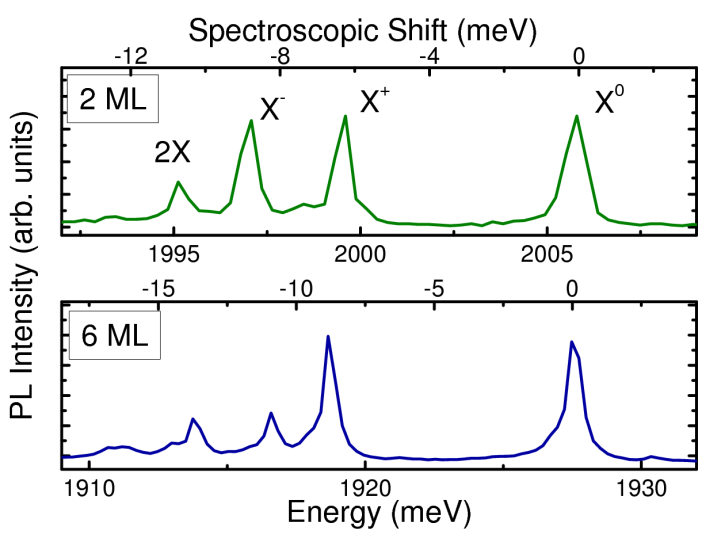

Fig. 1. PL spectra of two single QDs grown from $2 \mathrm{ML}$ (top) and 6 ML (bottom) thick CdTe layer. Top axis is relative to the $\mathrm{X}^{0} \mathrm{PL}$ energy.

In Fig. 2 we present spectroscopic shifts, taken as the $E_{\chi}-E_{\mathrm{X}^{0}}$, for 12 different QDs, grown at three different MBE labs, all of them exhibiting the same transition sequence. The identification of the transitions is based on the comparison of the spectroscopic shifts with those reported in literature. In fact, whenever the charge state of a recombining complex was determined [46, 54, 57-61], either by photon correlation measurements [46, 57], by charging behavior in electric field [54,61], by optical anisotropy $[57,58]$ or optical orientation [60], the same transition sequence was observed. A study of 200 dots corroborating this observation was reported [62]. However, we cannot exclude the possibility of another transition sequence, especially for small dots emitting at high energies (see below).

The universal redshift of the recombinations of charged complexes stand in strong contrast to the spectroscopic shifts measured for self-assembled InGaAs dots in GaAs barriers, where both the redshift and blueshift of the $\mathrm{X}^{+}$ with respect to the $\mathrm{X}^{0}$ was observed [63]. Theoretically,

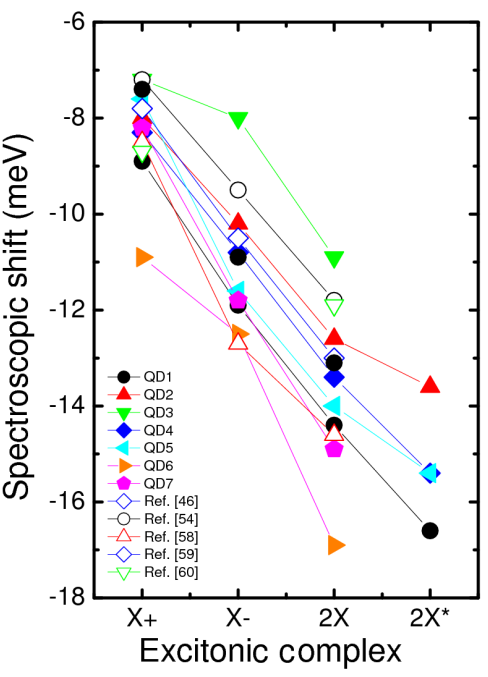

Fig. 2. Spectroscopic shifts of different excitonic complexes for seven QDs studied in this work and compared with five other reported in literature.

the sign of the spectroscopic shift is determined by the QD morphology, where In/Ga intermixing and dot height are crucial parameters [50]. Clearly, a qualitatively different situation is encountered in CdTe dots, where for all reported dots the transition sequence is the same [61, 62].

In order to explain the universal redshift of the charged exciton transitions, we describe the spectroscopic shifts separating the contributions of single particle Coulomb interactions, usually calculated in the framework of the Hartree-Fock (HF) approximation, from the Coulomb correlations resulting from the fact that electron and hole in-plane motions are not independent [64, 65], and from exchange energies important for multiply charged complexes to account for their fine structure $[12,16,66]$ and exchange induced band gap renormalization [67]. Under such approach, the $s$-shell emission energy of a complex containing $N_{\mathrm{e}}$ electrons and $N_{\mathrm{h}}$ holes, reads

$$
\begin{aligned}
& E\left(N_{\mathrm{e}}, N_{\mathrm{h}}\right)=E_{\mathrm{e} 1}+E_{\mathrm{h} 1}-J_{\mathrm{eh}} \\
& +\sum_{\mathrm{e}}\left(J_{\mathrm{ee}}-J_{\mathrm{eh}}\right)+\sum_{\mathrm{h}}\left(J_{\mathrm{hh}}-J_{\mathrm{eh}}\right) \\
& +\Delta_{\mathrm{corr}}\left(N_{\mathrm{e}}, N_{\mathrm{h}}\right)+\Delta_{\mathrm{exch}}\left(N_{\mathrm{e}}, N_{\mathrm{h}}\right),
\end{aligned}
$$

where $E_{\mathrm{e} 1}, E_{\mathrm{h} 1}$ are single particle electron and hole ground state energies,

$$
J_{i j}=e^{2} \int \frac{\left|\Psi\left(\boldsymbol{r}_{i}\right)\right|^{2}\left|\Psi\left(\boldsymbol{r}_{j}\right)\right|^{2}}{\varepsilon\left|\boldsymbol{r}_{i}-\boldsymbol{r}_{j}\right|} \mathrm{d} \boldsymbol{r}_{i} \mathrm{~d} \boldsymbol{r}_{j}
$$

is a Coulomb integral describing a direct interaction between particle $i$ and particle $j$ and $\Delta_{\text {corr }}, \Delta_{\text {exch }}$ are correlation and exchange energies, respectively. Thus, the first line is the emission energy of a single electron-hole pair (a neutral exciton), the second presents HF energies resulting from the interactions between remaining carriers in the complex, and the third and fourth lines are, respectively, correlation and exchange corrections, both 
of them negative [65]. In the following, we shall neglect the exchange corrections, since we are only interested in the four transitions shown in Fig. 1.

The contributions of the HF terms and correlation energies are schematically depicted in Fig. 3. The top curve represents a spectrum of the neutral exciton. If only the $\mathrm{HF}$ energies were to be taken into account, only single particle electron and hole wave functions would be important. Then, spectroscopic shifts for the $\mathrm{X}^{+}$and $\mathrm{X}^{-}$ are given by [68]:

$$
\begin{aligned}
& E_{\mathrm{X}^{+}}-E_{\mathrm{X}^{0}}=J_{\mathrm{ee}}-J_{\mathrm{eh}}, \\
& E_{\mathrm{X}^{-}}-E_{\mathrm{X}^{0}}=J_{\mathrm{hh}}-J_{\mathrm{eh}} .
\end{aligned}
$$

If we assume that the wave function of a hole, due to a larger effective mass, is spatially less extended than that of an electron, then $J_{\mathrm{hh}}>J_{\mathrm{eh}}>J_{\mathrm{ee}}$ and we find the $\mathrm{X}^{+}$ transition blueshifted and $\mathrm{X}^{-}$redshifted with respect to the $\mathrm{X}^{0}$ as depicted in the middle spectrum in Fig. 3. Such

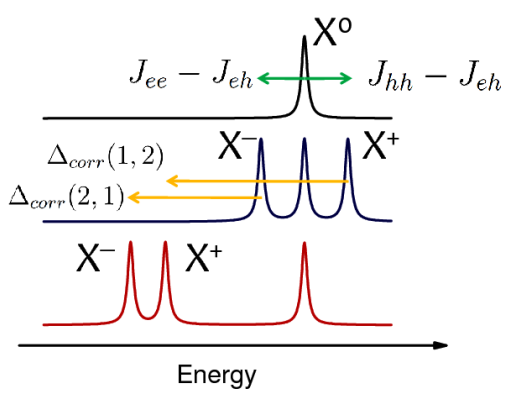

Fig. 3. A scheme showing different contributions to charged exciton spectroscopic shifts (confront Eq. (1) and see text). Top: $\mathrm{X}^{0}$ PL spectrum. Middle: $\mathrm{X}^{0}$, $\mathrm{X}^{+}$, and $\mathrm{X}^{-}$spectra modeled by only including single particle contributions. Bottom: spectrum modeled by taking into account both single particle and correlation contributions.

a transition sequence is reported for a majority of self-assembled InGaAs dots [15, 29, 63, 65, 69, 70], proving that in this system the confinement, i.e., the shape of the single particle wave functions, is the main contribution to the spectroscopic shifts. When we include the correlation energies, both the $\mathrm{X}^{+}$and $\mathrm{X}^{-}$redshift. If the correlations dominate over the direct Coulomb contributions, both of these transitions end up on the low energy side of the $\mathrm{X}^{0}$ as shown in the bottom spectrum in Fig. 3. Therefore, we conclude that in CdTe QDs the Coulomb correlations dominate over the contributions related solely to confinement. These correlations can be described in the language of configuration mixing (or configuration interaction, CI): the Coulomb interaction induces mixing of different excitonic configurations related to occupancies of more than one electron and hole shell [64, 71, 72]. We will discuss the details of the configuration mixing below, when assessing the lifetimes and Stark shifts of charged exciton transitions.

We remark that the redshift of both charged exciton transitions with respect to the $\mathrm{X}^{0}$ has also been observed for GaAs QDs fabricated by monolayer well width fluctuations [73]. In fact, the same transition sequence where $E_{\mathrm{X}^{0}}>E_{\mathrm{X}^{+}}>E_{\mathrm{X}^{-}}$was reported for more than 30 dots with different morphologies. These dots are substantially larger than self-assembled, epitaxial InGaAs dots so in the following we will intend to relate the size with the impact of the Coulomb correlations on spectroscopic properties of CdTe dots.

\section{Recombination rates}

Measurements of recombination rates yield additional information on the wave functions of carriers and excitons. In particular, they allow to assess the nature of the excitonic confinement and variations of single carrier wave functions upon changing the dot occupancy [63, 64]. In the strong confinement limit (SCL), where the excitonic Bohr radius is much larger than the size of a dot, the form of the wave functions is determined only by the QD morphology and very weakly modified by the Coulomb interactions. Hence, the recombination rates for single neutral and charged excitons are independent of the charge state and the biexciton decay is twice as fast as the single exciton decay, since the $2 \mathrm{X}$ has two decay channels, while $\mathrm{X}^{0}$ has only one. In this case, we can treat the wave functions as stiff and the Coulomb interactions as a perturbation to the QD confinement. For the SCL, the decay rate within a two-level model is given by [75]:

$$
\tau=\frac{3 \lambda_{\mathrm{PL}}^{2} \epsilon_{0} c m_{0}}{2 \pi n e^{2} f},
$$

where $\lambda_{\mathrm{PL}}$ is the $\mathrm{PL}$ wavelength, $n$ is the refractive index of the medium surrounding the $\operatorname{dot}(\mathrm{ZnTe})$ and $f$ the oscillator strength proportional to the overlap inte$\operatorname{gral}\left\langle\phi_{\mathrm{e}} \mid \phi_{\mathrm{h}}\right\rangle[76]$ :

$$
f=\left|\left\langle\phi_{\mathrm{e}} \mid \phi_{\mathrm{h}}\right\rangle\right|^{2} \frac{E_{\mathrm{P}}}{2 E_{\mathrm{PL}}},
$$

where $E_{\mathrm{PL}}$ is the PL photon energy and $E_{\mathrm{P}}=17.9 \mathrm{eV}$ is the Kane energy for CdTe and in the strong confinement limit $\left\langle\phi_{\mathrm{e}} \mid \phi_{\mathrm{h}}\right\rangle=1$. The above formula yields for a CdTe QD in a ZnTe matrix $(n=3.0)$ emitting at $2.0 \mathrm{eV}$ a decay rate of $0.77 \mathrm{~ns}^{-1}$.

In Fig. 4a, we show a map of a single dot PL signal time-resolved with a streak camera with an overall resolution of about 10 ps. The excitation source is a frequency doubled output of an optical parametric oscillator (providing an excitation wavelength of $532 \mathrm{~nm}$ ) pumped with a Ti:sapphire 2 ps laser. Figure $4 \mathrm{~b}$ presents a time-integrated spectrum exhibiting the familiar four transitions (confront with Fig. 1). Figure 4c shows the temporal profile of the $\mathrm{X}^{0}$ transition together with a fitted monoexponential decay. The fitted decay is offset with a constant value to account for the dark counts as well as a very long (several ns) decay associated with carrier recapture and/or dark exciton recombination. In order to draw robust conclusions, we analyze temporal decays from 6 different dots. We extract the recombi- 


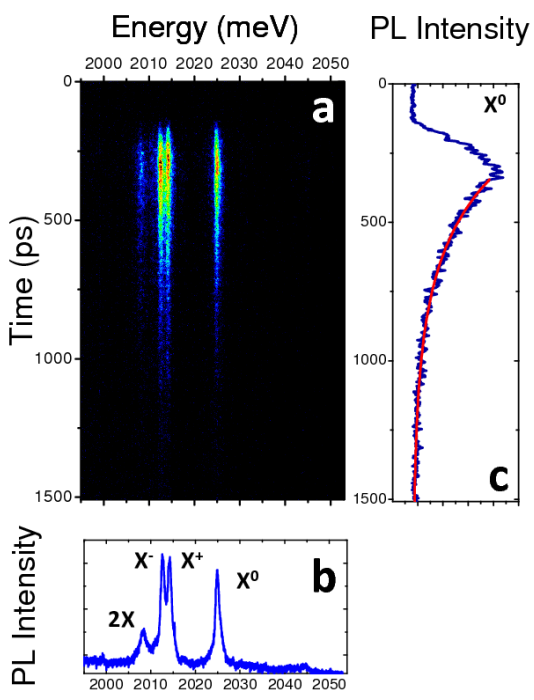

Fig. 4. (a) Time-resolved PL map of a single QD signal. (b) Single dot PL spectrum time-integrated from the map in (a). (c) Temporal profile of $\mathrm{X}^{0}$ recombination from (a).

Comparison of Bohr radii and dot lateral sizes for three systems discussed in the text.

\begin{tabular}{c|c|c|c|c}
\hline \hline System & Bohr & Lateral diameter & Decay rate \\
$\Gamma_{\mathrm{X}^{0}}$ & $L$ & $\Gamma_{\mathrm{SCL}}$ \\
\hline self-assembled InAs QDs & $13 \mathrm{~nm}$ & $5<L<15 \mathrm{~nm}[20,63]$ & $0.67<\Gamma_{\mathrm{X}^{0}}<1.25 \mathrm{~ns}^{-1}[63,78]$ & $0.87 \mathrm{~ns}^{-1}[63]$ \\
self-assembled CdTe QDs & $3.5 \mathrm{~nm}$ & $10<L<20 \mathrm{~nm}[37]$ & $4.3 \mathrm{~ns}^{-1}$ & $0.77 \mathrm{~ns}^{-1}$ \\
"natural" GaAs QDs & $13 \mathrm{~nm}$ & $20 \mathrm{~nm}<L[73]$ & $20<\Gamma_{\mathrm{X}^{0}}<40 \mathrm{~ns}^{-1}[79]$ & $1.0 \mathrm{~ns}^{-1}$
\end{tabular}

Even a superficial inspection of Fig. 4 suggests that $\Gamma$ depends on the QD charge state. In Fig. 5, we plot the extracted decay rates for charged excitons and biexciton transition as well as a transition slightly redshifted with respect to $2 \mathrm{X}$, which we tentatively ascribe to charged biexciton recombination [62] and label as $2 \mathrm{X}^{\star}$. The decay rates are given in units of the $\Gamma_{\mathrm{X}^{0}}$ given in the legend. It is clear that the decay rate depends on the QD occupancy. In particular, we find that on average $\Gamma_{\mathrm{X}^{+}} / \Gamma_{\mathrm{X}^{0}}=0.85 \pm 0.04$ and $\Gamma_{\mathrm{X}^{-}} / \Gamma_{\mathrm{X}^{0}}=0.96 \pm 0.04$. Measured decay rates $\Gamma$ are compared with decay rates expected for strong confinement limit $\Gamma_{\mathrm{SCL}}$. Thus, the recombination rate is decreased for the $\mathrm{X}^{+}$and almost unaffected for the $\mathrm{X}^{-}$. For the biexciton transitions, $\Gamma_{2 \mathrm{X}} / \Gamma_{\mathrm{X}^{0}}=1.37 \pm 0.09$ and $\Gamma_{2 \mathrm{X}^{\star}} / \Gamma_{\mathrm{X}^{0}}=1.39 \pm 0.15$ both substantially slower than what is expected for the SCL, where $\Gamma_{2 \mathrm{X}} / \Gamma_{\mathrm{X}^{0}}=2$.

The above observations indicate that for a dot occupied with a neutral exciton, the charging with a second hole results in stronger wave function modification than nation rates $\Gamma$ by fitting the monoexponential decays as shown in Fig. 4c. For the case of the $\mathrm{X}^{0}$, the mean decay rate $\Gamma_{\mathrm{X}^{0}}=4.3 \mathrm{~ns}^{-1}$, which is more than a factor of 5 larger than what we evaluate for the SCL. It is a direct indication that the confinement conditions in CdTe QDs are far from strong confinement limit.

In Table I, we compare excitonic Bohr radii with dot sizes for three systems: self-assembled InAs and CdTe dots and "natural" GaAs dots [77]. Only for the first system, the Bohr radius is comparable to the dot lateral diameter. Consequently, in these dots the recombination rates are relatively close to the values expected for the SCL $[63,78]$. Totally different situation is encountered for CdTe and "natural" GaAs dots. In the former case the Bohr radius is about $3.5 \mathrm{~nm}$ much smaller than a typical lateral self-assembled QD size. In the latter case, the lateral size is very large, with diameters larger than $20 \mathrm{~nm}$ owing to the fabrication procedures of the "natural" dots [73]. In both of these cases, the recombination rates are substantially larger than the ones evaluated for the SCL using Eq. (3). We therefore conclude that, in terms of confinement conditions, self assembled CdTe dots resemble rather the "natural" GaAs QDs than InAs self assembled dots - they are far from the strong confinement limit.

TABLE I

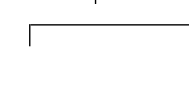


creased recombination rate suggests that the correlations admix a significant amount of optically inactive, dark configurations to the $\mathrm{X}^{+}$wave function. The noticeable decrease of the $\Gamma_{\mathrm{X}}+$ decay rate and a weaker change of the $\Gamma_{\mathrm{X}^{-}}$with respect to the $\Gamma_{\mathrm{X}^{0}}$ suggests that admixtures involving higher shell hole states are much stronger than admixtures of higher electronic states. We therefore conclude that the hole wave function is rather soft, while the electron wave function is rather stiff. These conclusions are correlated with the confinement conditions in the valence and conduction bands, respectively. In the $\mathrm{CdTe} / \mathrm{ZnTe}$ system, the valence band offset is negligible and the hole confinement stems mostly from the strain. Shallow confinement facilitates the Coulomb correlations by admixtures of higher shell states. Conversely, since almost all of the band offset produces the conduction band confinement, the electrons are rather strongly confined. Due to their smaller mass, the electronic shells are further separated than hole shells and mixing of different electronic configurations is not as important as for the holes.

We remark that recombination rates for different charge complexes in a CdTe QD have recently been calculated within an effective mass approximation, including the configuration mixing [80]. The model correctly predicted the decay rates, however, was not able to reproduce the magnitudes of the spectroscopic shifts, possibly due to not enough number of shells taken into consideration when constructing the correlated wave functions. We also note that analogous conclusions regarding the different importance of the Coulomb correlations for conduction and valence bands were reached for the InGaAs dots despite entirely different potential depths and dielectric constants [63, 64].

\section{Charge tuning and Stark effect}

In order to gain more insight into the electron and hole wave functions and the charge redistributions upon changing the dot occupancy, we perform PL measurements for dots in electric field. The dots are embedded in either a field-effect $p$-i-Schottky structure or a $n-i-p$ diode. Details of the sample structures are depicted in the top row of Fig. 6 . In the former structure, the back contact is a $p$-type ZnTe layer and on top a semitransparent $\mathrm{Ni} / \mathrm{Au}$ Schottky gate is evaporated [61]. The design of this structure is analogous to those routinely employed to control the charge state of InGaAs dots [12, 14, 15, 20, $29,63,69]$ via the Coulomb blockade effect described in Introduction. In the latter structure, a dot layer is embedded between an iodine doped $n$-type CdTe layer on the bottom and a nitrogen doped $p$-type ZnTe on top. In this structure the charge can be tuned by taking advantage of different capture rates for electrons and holes and their dependences on electric field. Before discussing the effect of the electric field on electron and hole wave functions, we will address the efficiency of charge control in the two types of structures.

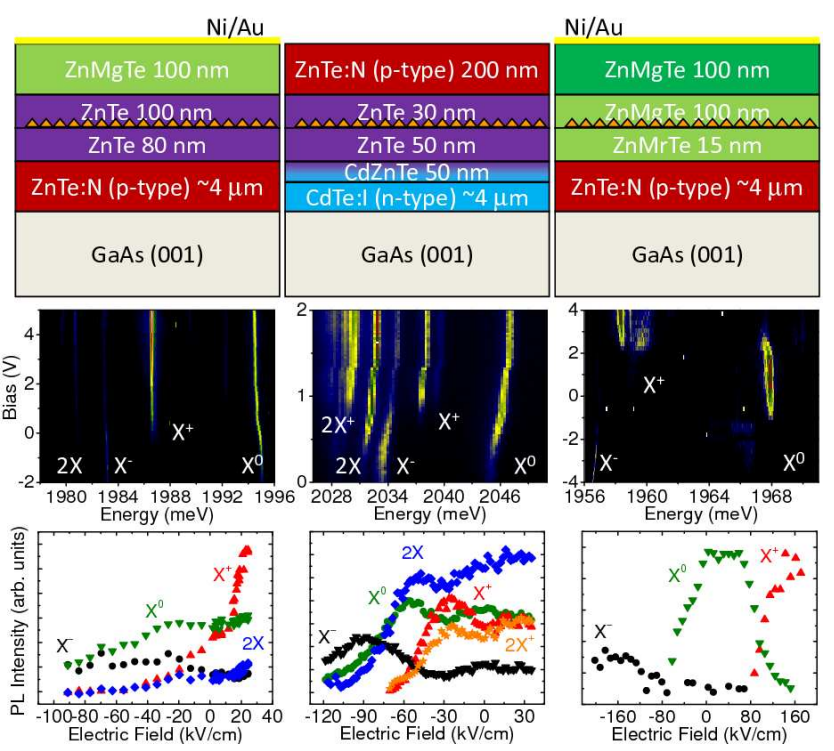

Fig. 6. Charging behavior for three structures discussed in the text. Left column: $p$ - $i$-Schottky structure with CdTe QDs in ZnTe barriers. Middle column: $n-i-p$ diode structure with CdTe QDs in ZnTe barriers. Right column: $p-i$-Schottky structure with CdTe QDs in $\mathrm{Zn}_{0.9} \mathrm{Mg}_{0.1}$ Te barriers. First row: sample structure. Second row: PL maps as a function of bias. Third row: PL intensity dependence on electric field.

In the left column of Fig. 6, we show the results for a QD in a $p-i$-Schottky structure depicted schematically on top left of the figure. The map in the middle left presents the bias dependence of the PL spectra. At negative (reverse) bias, the $\mathrm{X}^{-}$and $\mathrm{X}^{0}$ transitions are seen. As the bias is increased, the $\mathrm{X}^{-}$loses strength, while $\mathrm{X}^{+}$and $2 \mathrm{X}$ appear and eventually the $\mathrm{X}^{+}$dominates the spectrum at positive (forward) bias. The intensities of the four transitions are presented in the bottom graph as a function of the electric field $F=\left(U-U_{\mathrm{bi}}\right) / w$, where $w$ is the width of the intrinsic region and $U$ and $U_{\mathrm{bi}}$ are applied and built-in voltages, respectively. For forward bias, the electric field is screened by the current flow and therefore we use the curvature of the $\mathrm{X}^{0}$ Stark shift to calibrate the electric field [61]. It is clear that charge tunability is very limited in this structure as different charge states coexist in a large range of electric fields. The reason for this behavior is a wide spacer layer between the hole reservoir and the QD. Its width is $80 \mathrm{~nm}$, which makes the tunnel coupling relatively weak. Moreover, the excitation wavelength of $532 \mathrm{~nm}$ results in creation of electron-hole pairs above the dot excited states [55] and hence their separate trapping by the QD potential. Apparently and expectedly for such a wide barrier, the tunneling rate is slower than the capture rate, which results in a coexistence of charge states [61, 81].

A different situation is encountered for the $n-i-p$ structure presented in the top middle picture of Fig. 6. The spacer between the dots and the $n$-type CdTe consists of 
a $50 \mathrm{~nm}$ thick $\mathrm{Cd}_{1-x} \mathrm{Zn}_{x}$ Te layer with a gradient of zinc fraction $x$ along the growth axis and a $50 \mathrm{~nm}$ thick ZnTe layer. The map of the PL bias dependence presented in Fig. 6 (middle) also shows a coexistence of charge states, but analysis of the PL intensities (middle bottom) clearly show ranges of electric field, where one of the charge states dominates. Namely, for $F<-90 \mathrm{kV} / \mathrm{cm}$, the dot is charged with a single electron and the $\mathrm{X}^{-}$ recombination is the strongest transition. The domination of the $\mathrm{X}^{-}$occurs as a result of enhanced hole tunneling out of the dot under reverse bias [82]. For $-90<F<-40 \mathrm{kV} / \mathrm{cm}$, the hole tunneling is slowed down, the dot is neutral, and the $\mathrm{X}^{0}$ and $2 \mathrm{X}$ dominate the spectrum. Above $-40 \mathrm{kV} / \mathrm{cm}$, the intensities of neutral complexes decrease, while the $\mathrm{X}^{+}$intensity increases. However, the $\mathrm{X}^{+}$intensity is still lower than that of the $2 \mathrm{X}$. This is probably due to a large excitation density enabling capture of two electron-hole pairs before one of them recombines. Indeed, at low energy side of the 2X transition another transition appears simultaneously with the $\mathrm{X}^{+}$and has almost identical intensity dependence on electric field. We identify it therefore as a positively charged biexciton $2 \mathrm{X}^{+}$.

The best performance in terms of charge tunability is obtained for the structure depicted in the right column of Fig. 6. In this case, the dots are grown in $\mathrm{Zn}_{0.9} \mathrm{Mg}_{0.1} \mathrm{Te}$ barriers to enhance the confinement in the valence band. As a result, the charge fluctuations are suppressed and clear charging steps are observed. Moreover, the tunnel spacer layer in this sample is $15 \mathrm{~nm}$, which greatly increases the tunneling rate for holes from the back contact to the dots. The intensity dependence on $F$ clearly shows that at $F<-80 \mathrm{kV} / \mathrm{cm}$ the dot is negatively charged, neutral for $-80<F<100 \mathrm{kV} / \mathrm{cm}$ and positively charged for $F>100 \mathrm{kV} / \mathrm{cm}$.

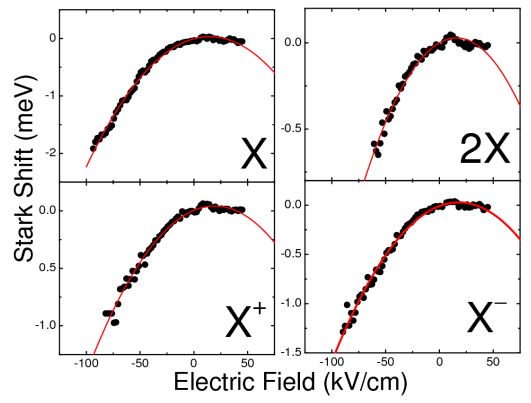

Fig. 7. Stark shifts for $\mathrm{X}^{0}, \mathrm{X}^{+}, \mathrm{X}^{-}$, and $2 \mathrm{X}$ transitions with fitted second order polynomials according to Eq. (5).

The electric field not only leads to changes in QD occupancies, but also shifts the transition energies due to the QCSE. For moderate fields, the transition energies are well approximated by the second order of the perturbation expansion and given by

$$
E(F)=E(0)-p F+\beta F^{2},
$$

where $p$ and $\beta$ are the built-in dipole moment and electron-hole polarizability, respectively $[19,20]$. The former value yields a distance between the centers of gravity of electron and hole wave functions under no electric field and the latter evaluates, how easily they can be pulled apart. Fitting of Eq. (5) to the Stark shifts of $\mathrm{X}^{0}$, $\mathrm{X}^{+}, \mathrm{X}^{-}$, and $2 \mathrm{X}$ transitions for the dot embedded in a $n-i-p$ structure is presented in Fig. 7 .

Polarizability obtained from the fit for the $\mathrm{X}^{0}$ transition is $\beta / e=17.3 \pm 0.4 \mathrm{~nm}^{2} / \mathrm{V}$. For the dot presented in the left column of Fig. 6 , we obtained $\beta / e=$ $5.7 \pm 0.9 \mathrm{~nm}^{2} / \mathrm{V}[61]$. These values are substantially smaller than $\beta \approx 40 \mathrm{~nm}^{2} / \mathrm{V}$ reported for InAs dots [20] pointing to stronger Coulomb attraction between the electron and the hole in the II-VI compound.

\section{TABLE II}

Values of built-in dipole moment $p$ obtained for different excitonic complexes by fitting of Eq. (5) to the transition energies dependence on electric field as shown in Fig. 7. The reduction of $p$ relative to the $\mathrm{X}^{0}$ is demonstrated.

\begin{tabular}{c|c|c|c|c}
\hline \hline \multirow{2}{*}{} & \multicolumn{2}{|c|}{$p-i$-Schottky [61] } & \multicolumn{2}{c}{$n-i-p$} \\
\cline { 2 - 5 } & $p / e[\AA]$ & $\Delta p / p_{\mathrm{X}^{0}}$ & $p / e[\AA]$ & $\Delta p / p_{\mathrm{X}^{0}}$ \\
\hline $\mathrm{X}^{0}$ & $0.89 \pm 0.03$ & - & $0.50 \pm 0.02$ & - \\
$\mathrm{X}^{+}$ & $0.08 \pm 0.02$ & $-91 \%$ & $0.39 \pm 0.02$ & $-22 \%$ \\
$\mathrm{X}^{-}$ & $0.45 \pm 0.02$ & $-49 \%$ & $0.42 \pm 0.02$ & $-8 \%$ \\
$2 \mathrm{X}$ & $0.48 \pm 0.01$ & $-46 \%$ & $0.36 \pm 0.01$ & $-28 \%$
\end{tabular}

Obtained values of the built-in dipole $p$ together with a reduction relative to the value for the $\mathrm{X}^{0}$ are presented in Table II for the dots in the left and middle columns of Fig. 6. The built-in dipole evaluated from fitting in Fig. 7 is $p_{\mathrm{X}^{0}} / e=+0.50 \pm 0.02 \AA$, while for the dot in the left column of Fig. 6 we obtained $p_{\mathrm{X}^{0}} / e=+0.89 \pm 0.03 \AA$. For both of the dots, the sign of $p$ is positive, indicating that under zero electric field, looking along the growth axis, the hole is located above the electron. This alignment is contrary to what is expected for a pure CdTe dot in a ZnTe barrier. Obviously, for a dot with a translational symmetry along the growth axis, no dipole moment is expected. For a lens shaped dot, along the growth axis the lateral size decreases and hence the in-plane potential well becomes shallower towards the apex of the dot. One would expect therefore that the hole, as a heavier particle, would localize closer to the QD base, while the electron would remain closer to the top. This is clearly not the case implying that the dot is not homogeneous along the growth axis. Indeed, such inverted carrier alignment was also observed in InAs dots [11] and interpreted as resulting from In/Ga intermixing along the growth axis [19]. We therefore suggest that $\mathrm{Cd} / \mathrm{Zn}$ intermixing is responsible for the positive built-in dipoles in the CdTe dots discussed here. We remark however that, similarly to the InAs dot [20], the magnitude of $p$ 
depends on the emission energy, thus on the dot size, indicating again a strong sensitivity of the dipole value to QD morphology.

The value of the dipole moment decreases when carriers are added to a dot occupied by a single exciton (see Table II). This effect is a direct consequence of wave function redistributions upon changing the dot occupancy. We find that upon addition of an extra hole, the decrease in $p$ is stronger than when adding a second electron. This is another indication of the hole wave function being softer than electron wave function as pointed out in the discussion of recombination rates.

\section{Conclusions and outlook}

Our results show that the Coulomb correlations in self-assembled CdTe QDs are the major factors determining the optical spectra and recombination rates. In all the studied dots (and in those reported by other groups) the transition sequence is the same underlying the robustness of the correlation effects. Namely, charged exciton recombinations are always redshifted with respect to the neutral exciton, in stark contrast with the InGaAs QDs, where redshifts and blueshifts were reported. Also, enhanced Coulomb correlations result in an increase of the recombination rate with respect to the strong confinement limit, where correlations are absent. We find that the rates in CdTe QDs are over a factor of 5 larger than what is expected for the strong confinement limit. When the confinement is relatively weak, the exciton can be viewed as a quasi-free particle moving in the QD potential and picking up radiative contributions from each elementary cell [63]. As a result, we deal with a sort of a superradiant effect, which leads to accelerated emission. Analogous conclusions regarding the importance of correlation effects can be reached for "natural" GaAs QDs. In that case, the charged exciton recombinations are also always on the low energy side of the neutral exciton [73] and the acceleration of the recombination is even more pronounced (see Table I). We therefore conclude that the confinement conditions in CdTe QDs resemble rather the "natural" GaAs dots than the self-assembled InGaAs system.

We remark that CdTe QDs form a very inhomogeneous ensemble and therefore we cannot exclude a possibility of finding dots with a much stronger confinement, where both the transition sequence and recombinations rates would be different. We would expect such dots on the high energy side of the ensemble emission band and fabricated from a relatively wide CdTe layer, which was shown to decrease the lateral size [49]. For such dots, we would expect a transition sequence similar to the one reported for a majority of InGaAs dots, with the $\mathrm{X}^{+}$blueshifted with respect to the $\mathrm{X}^{0}$. As for the decay rates, the radiative decay rate should be much smaller compared to the dots described here. However, since the transition energy of such dots would be on the high energy side of the ensemble PL band, probably the observed decays would be shortened due to excitation transfer between the dots [41].

We demonstrate that the recombination rates depend on the QD charge state. We find that on average the emission rate is decreased upon addition of an extra hole, while adding an extra electron result in a much smaller effect. We thus conclude that the hole wave function undergoes a stronger redistribution upon charging than electron wave function. We support this conclusion with a measurement of a built-in dipole moment, which provides mean electron-hole distance in a dot. We evaluate the built-in dipole from analysis of the Stark shifts of QD transitions. We find that the value of the built-in dipole is importantly reduced upon addition of an extra hole, while weakly modified when charging with an extra electron. Therefore, the analysis of the decay rates and built-in dipole reductions lead us to a conclusion that the hole wave function is rather soft, while the electron wave function is rather stiff. These findings are related to the confinement conditions in valence and conduction bands, respectively. Owing to a small $\mathrm{CdTe} / \mathrm{ZnTe}$ valence band offset, the hole confinement is rather weak. On the other hand, almost all the band offset produces the confinement for the electrons, which as a result are confined more strongly. Weak confinement facilitates configuration mixing, which on the one hand results in a decrease of the recombination rates (by admixtures of optically inactive configurations) and on the other hand leads to a decrease of the built-in dipole.

In order to provide tunable charging of a dot, we fabricate field effect or diode structures with dots embedded in the intrinsic region. We employ three different approaches: CdTe dots in ZnTe barriers in $p$ - $i$-Schottky or $n-i-p$ structures and CdTe dots in a $\mathrm{Zn}_{1-x} \mathrm{Mg}_{x}$ Te barrier with increased valence band confinement. We found that this last structure provided clearest charging steps however a detailed study of the influence of the width of the spacer layer separating the dots from $p$-type layer in the $p$ - $i$-Schottky structure is necessary.

We hope that our studies of charging effects in CdTe QDs will become a starting point to other, more sophisticated investigations. In particular, we believe that evaluation of hyperfine interaction strength deserves immediate attention. As mentioned in Introduction, a substantially weaker nuclei-induced qubit dephasing is expected as a result of much smaller abundances of non-zero spin isotopes. Nuclear polarization can be created by either pumping a single carrier with a well defined spin, or by separating the photocarriers by electric field to keep them from recombining as was already shown [82].

Another important goal is to demonstrate electrical control of magnetization in $\mathrm{Cd}_{1-x} \mathrm{Mn}_{x}$ Te QDs. We have shown how to monitor the formation of the magnetization in single dots [33]. It is now necessary to perform an analogous experiment under conditions of controllable charging and observe changes in formation dynamics. Such an experiment should also provide new data on charged excitons in these multiply Mn-doped QDs. 
Although charge control of a single Mn-doped CdTe QD has already been demonstrated [54], it would be very interesting to apply it to studies of the orientation dynamics of the Mn spin. Apart from clear benefits from the basic research standpoint, the data could enable optimization of information storage for potential devices.

Any coherent control of a QD qubit requires a resonant (or quasi-resonant) selective excitation of the neutral exciton $[4,5]$. Dots embedded in field effect structures make possible a readout of the qubit state without blinding the detector when measuring resonantly excited PL. Namely, by measuring the photocurrent it is possible to perform even two-qubit operations [23]. We hope that our studies of electron storage [82] will lay ground for the experiments involving qubit operations in CdTe QDs.

As pointed out in Introduction, growth of vertically stacked CdTe dots is feasible. It is therefore possible to study electronic coupling in two layers of dots and demonstrate formation of a quantum dot molecule. Such objects have already been studied in the InGaAs system [83] and the coupling was understood in terms of single carrier tunneling. Due to stronger Coulomb correlations in CdTe dots, here the coupling schemes could be very different. In particular, it would be important to assess whether a long range coupling could be present. It was shown for CdSe dots that such long-range interaction can lead to a superradiant effect [84]. Different coupling mechanisms could possibly lead to new strategies in applications of CdTe dots as quantum gates. Combining the coupling within a QD molecule with giant Zeeman effect of Mn-doped QDs should provide a possibility to control the coupling by magnetic field.

\section{Acknowledgments}

The author wishes to thank V. Voliotis, A. Kudelski, O. Krebs, A.I. Tartakovskii, M.S. Skolnick, P. Wojnar, K. Fronc, P. Rutkowski, M. Korkusiński, P. Hawrylak, Ł. Cywiński, G. Karczewski, and T. Wojtowicz. This research was supported by a Polish Ministry of Science and Education grant no. 0634/BH03/2007/33 and the Polonium Programme and by European Union within European Regional Development Fund, through Innovative Economy grant (POIG.01.01.02-00-008/08) and SANDiE Network of Excellence.

\section{References}

[1] A. Barenco, D. Deutsch, A. Ekert, R. Jozsa, Phys. Rev. Lett. 74, 4083 (1995).

[2] Single Semiconductor Quantum Dots, Ed. P. Michler, Springer-Verlag, Berlin 2009.

[3] E. Peter, P. Senellart, D. Martrou, A. Lemaître, J. Hours, J.M. Gérard, J. Bloch, Phys. Rev. Lett. 95, 067401 (2005).

[4] N.H. Bonadeo, J. Erland, D. Gammon, D. Park, D.S. Katzer, D.G. Steel, Science 282, 1473 (1998).

[5] H. Kamada, H. Gotoh, J. Temmyo, T. Takagahara, H. Ando, Phys. Rev. Lett. 87, 24640 (2001).
[6] L. Besombes, J.J. Baumberg, J. Motohisa, Phys. Rev. Lett. 90, 257402 (2003).

[7] P. Michler, A. Kiraz, C. Becher, W.V. Schoenfeld, P.M. Petroff, L. Zhang, E. Hu, A. Imamoglu, Science 290, 2292 (2000).

[8] R.M. Stevenson, R.J. Young, P. Atkinson, K. Cooper, D.A. Ritchie, A.J. Shields, Nature 439, 179 (2005).

[9] N. Akopian, N.H. Lindner, E. Poem, Y. Berlatzky, J. Avron, D. Gershoni, B.D. Gerardot, P.M. Petroff, Phys. Rev. Lett. 96, 130501 (2006).

[10] H. Drexler, D. Leonard, W. Hansen, J.P. Kotthaus, P.M. Petroff, Phys. Rev. Lett. 73, 224 (1994).

[11] P.W. Fry, I.E. Itskevich, D.J. Mowbray, M.S. Skolnick, J.J. Finley, J.A. Barker, E.P. O'Reilly, L.R. Wilson, I.A. Larkin, P.A. Maksym, M. Hopkinson, M. Al-Khafaji, J.P.R. David, A.G. Cullis, G. Hill, J.C. Clark, Phys. Rev. Lett. 84, 733 (2000).

[12] R.J. Warburton, C. Schäflein, D. Haft, F. Bickel, A. Lorke, K. Karrai, J.M. Garcia, W. Schoenfeld, P.M. Petroff, Nature 405, 926 (2000).

[13] S. Tarucha, D.G. Austing, T. Honda, R.J. van der Hage, L.P. Kouwenhoven, Phys. Rev. Lett. 77, 3613 (1996).

[14] M. Ediger, K. Karrai, A. Badolato, P.M. Petroff, R.J. Warburton, Phys. Status Solidi C 3, 3806 (2006).

[15] M. Ediger, G. Bester, A. Badolato, P.M. Petroff, K. Karrai, A. Zunger, R.J. Warburton, Nature Phys. 3, 774 (2007).

[16] L. He, G. Bester, A. Zunger, Phys. Rev. Lett. 95 , 246804 (2005).

[17] S.A. Empedocles, M.G. Bawendi, Science 278, 2114 (1997).

[18] S. Raymond, J.P. Reynolds, J.L. Merz, S. Fafard, Y. Feng, S. Charbonneau, Phys. Rev. B 58, R13415 (1998).

[19] J.A. Barker, E.P. O'Reilly, Phys. Rev. B 61, 13840 (2000).

[20] J.J. Finley, M. Sabathil, P. Vogl, G. Abstreiter, R. Oulton, A.I. Tartakovskii, D.J. Mowbray, M.S. Skolnick, S.L. Liew, A.G. Cullis, M. Hopkinson, Phys. Rev. B 70, 201308 (2004).

[21] A. Zrenner, E. Beham, S. Stufler, F. Findeis, M. Bichler, G. Abstreiter, Nature 418, 612 (2002).

[22] S. Stufler, P. Ester, A. Zrenner, M. Bichler, Phys. Rev. B 72, 121301 (2005).

[23] A.J. Ramsay, S.J. Boyle, R.S. Kolodka, J.B.B. Oliveira, J. Skiba-Szymanska, H.Y. Liu, M. Hopkinson, A.M. Fox, M.S. Skolnick, Phys. Rev. Lett. 100, 197401 (2008).

[24] S.J. Boyle, A.J. Ramsay, F. Bello, H.Y. Liu, M. Hopkinson, A.M. Fox, M.S. Skolnick, Phys. Rev. B 78, 075301 (2008).

[25] M. Kroutvar, Y. Ducommun, D. Heiss, M. Bichler, D. Schuh, G. Abstreiter, J.J. Finley, Nature 432, 81 (2004).

[26] D. Heiss, V. Jovanov, M. Bichler, G. Abstreiter, J.J. Finley, Phys. Rev. B 77, 235442 (2008).

[27] D. Gammon, A.L. Efros, T.A. Kennedy, M. Rosen, D.S. Katzer, D. Park, S.W. Brown, V.L. Korenev, I.A. Merkulov, Phys. Rev. Lett. 86, 5176 (2001). 
[28] I.A. Merkulov, A.L. Efros, M. Rosen, Phys. Rev. B 65, 205309 (2002).

[29] B. Eble, O. Krebs, A. Lemaitre, K. Kowalik, A. Kudelski, P. Voisin, B. Urbaszek, X. Marie, T. Amand, Phys. Rev. B 74, 081306 (2006).

[30] A.I. Tartakovskii, T. Wright, A. Russell, V.I. Fal'ko, A.B. Van'kov, J. Skiba-Szymanska, I. Drouzas, R.S. Kolodka, M.S. Skolnick, P.W. Fry, A. Tahraoui, H.-Y. Liu, M. Hopkinson, Phys. Rev. Lett. 98, 026806 (2007).

[31] O. Krebs, B. Eble, A. Lemaitre, P. Voisin, B. Urbaszek, T. Amand, X. Marie, C.R. Physique 9, 874 (2008).

[32] P. Wojnar, J. Suffczyński, K. Kowalik, A. Golnik, G. Karczewski, J. Kossut, Phys. Rev. B 75, 155301 (2007).

[33] L. Kłopotowski, L. Cywiński, P. Wojnar, V. Voliotis, K. Fronc, T. Kazimierczuk, A. Golnik, M. Ravaro, R. Grousson, G. Karczewski, T. Wojtowicz, Phys. Rev. B 83, 081306 (2011).

[34] J. Fernández-Rossier, L. Brey, Phys. Rev. Lett. 93 117201 (2004).

[35] A.O. Govorov, Phys. Rev. B 72, 075359 (2005).

[36] R.M. Abolfath, P. Hawrylak, I. Žutić, Phys. Rev. Lett. 98, 207203 (2007).

[37] F. Tinjod, B. Gilles, S. Moehl, K. Kheng, H. Mariette, Appl. Phys. Lett. 82, 4340 (2003).

[38] J. Kobak, W. Pacuski, T. Jakubczyk, T. Kazimierczuk, A. Golnik, K. Frank, A. Rosenauer, C. Kruse, D. Hommel, J. Gaj, Acta Phys. Pol. A 119, 627 (2011).

[39] G. Karczewski, S. Maćkowski, M. Kutrowski, T. Wojtowicz, J. Kossut, Appl. Phys. Lett. 74, 3011 (1999).

[40] T.A. Nguyen, S. Mackowski, H.E. Jackson, L.M. Smith, J. Wrobel, K. Fronc, G. Karczewski, J. Kossut, M. Dobrowolska, J.K. Furdyna, W. Heiss, Phys. Rev. B 70, 125306 (2004).

[41] S. Mackowski, G. Prechtl, W. Heiss, F.V. Kyrychenko, G. Karczewski, J. Kossut, Phys. Rev. B 69, 205325 (2004).

[42] Z. Wasilewski, S. Fafard, J. McCaffrey, J. Cryst. Growth 201/202, 1131 (1999).

[43] S. Mackowski, G. Karczewski, T. Wojtowicz, J. Kossut, S. Kret, A. Szczepańska, P. Dlużewski, G. Prechtl, W. Heiss, Appl. Phys. Lett. 78, 3884 (2001).

[44] L. Besombes, K. Kheng, L. Marsal, H. Mariette, Phys. Rev. B 63, 155307 (2001)

[45] R. Hanbury Brown, R.Q. Twiss, Nature 178, 4541 (1956).

[46] J. Suffczyński, T. Kazimierczuk, M. Goryca, B. Piechal, A. Trajnerowicz, K. Kowalik, P. Kossacki, A. Golnik, K.P. Korona, M. Nawrocki, J.A. Gaj, G. Karczewski, Phys. Rev. B 74, 085319 (2006).

[47] L. Marsal, L. Besombes, F. Tinjod, K. Kheng, A. Wasiela, B. Gilles, J.-L. Rouvière, H. Mariette, Appl. Phys. Lett. 91, 4936 (2002).

[48] D. Gammon, E.S. Snow, B.V. Shanabrook, D.S. Katzer, D. Park, Science 273, 87 (1996).
[49] K. Kukliński, Ł. Kłopotowski, K. Fronc, M. Wiater, P. Wojnar, P. Rutkowski, V. Voliotis, R. Grousson, G. Karczewski, J. Kossut, T. Wojtowicz, Appl. Phys. Lett. 99, 141906 (2011).

[50] L. Besombes, Y. Léger, L. Maingault, D. Ferrand, H. Mariette, J. Cibert, Phys. Rev. Lett. 93, 207403 (2004).

[51] L. Maingault, L. Besombes, Y. Leger, C. Bougerol, H. Mariette, Appl. Phys. Lett. 89, 193109 (2006).

[52] C. Le Gall, L. Besombes, H. Boukari, R. Kolodka, J. Cibert, H. Mariette, Phys. Rev. Lett. 102, 127402 (2009).

[53] M. Goryca, T. Kazimierczuk, M. Nawrocki, A. Golnik, J.A. Gaj, P. Kossacki, P. Wojnar, G. Karczewski, Phys. Rev. Lett. 103, 087401 (2009).

[54] Y. Léger, L. Besombes, J. Fernández-Rossier, L. Maingault, H. Mariette, Phys. Rev. Lett. 97, 107401 (2006).

[55] T.A. Nguyen, S. Mackowski, T.B. Hoang, H.E. Jackson, L.M. Smith, G. Karczewski, Phys. Rev. B $\mathbf{7 6}$, 245320 (2007).

[56] L. Besombes, K. Kheng, L. Marsal, H. Mariette, Phys. Rev. B 65, 121314 (2002).

[57] T. Kazimierczuk, M. Goryca, M. Koperski, A. Golnik, J.A. Gaj, M. Nawrocki, P. Wojnar, P. Kossacki, Phys. Rev. B 81, 155313 (2010).

[58] K. Kowalik, O. Krebs, P. Senellart, A. Lemaître, B. Eble, A. Kudelski, J.A. Gaj, P. Voisin, Phys. Status Solidi C 3, 3890 (2006).

[59] H.S. Lee, A. Rastelli, M. Benyoucef, F. Ding, T.W. Kim, H.L. Park, O.G. Schmidt, Nanotechnology 20, 075705 (2009).

[60] T. Kazimierczuk, J. Suffczynski, A. Golnik, J.A. Gaj, P. Kossacki, P. Wojnar, Phys. Rev. B 79, 153301 (2009).

[61] Ł. Kłopotowski, V. Voliotis, A. Kudelski, A.I. Tartakovskii, P. Wojnar, K. Fronc, R. Grousson, O. Krebs, M.S. Skolnick, G. Karczewski, T. Wojtowicz, Phys. Rev. B 83, 155319 (2011).

[62] T. Kazimierczuk, T. Smoleński, M. Goryca, L. Kłopotowski, P. Wojnar, K. Fronc, A. Golnik, M. Nawrocki, J.A. Gaj, P. Kossacki, Phys. Rev. B 84, 165319 (2011).

[63] P.A. Dalgarno, J.M. Smith, J. McFarlane, B.D. Gerardot, K. Karrai, A. Badolato, P.M. Petroff, R.J. Warburton, Phys. Rev. B 77, 245311 (2008).

[64] G.W. Bryant, Phys. Rev. B 37, 8763 (1988).

[65] D.V. Regelman, E. Dekel, D. Gershoni, E. Ehrenfreund, A.J. Williamson, J. Shumway, A. Zunger, W.V. Schoenfeld, P.M. Petroff, Phys. Rev. B 64, 165301 (2001).

[66] J.J. Finley, P.W. Fry, A.D. Ashmore, A. Lemaître, A.I. Tartakovskii, R. Oulton, D.J. Mowbray, M.S. Skolnick, M. Hopkinson, P.D. Buckle, P.A. Maksym, Phys. Rev. B 63, 161305 (2001).

[67] M. Bayer, O. Stern, P. Hawrylak, S. Fafard, A. Forchel, Nature 405, 923 (2000).

[68] G. Bester, A. Zunger, Phys. Rev. B 68, 073309 (2003). 
[69] M.E. Ware, E.A. Stinaff, D. Gammon, M.F. Doty, A.S. Bracker, D. Gershoni, V.L. Korenev, S.C. Bădescu, Y. Lyanda-Geller, T.L. Reinecke, Phys. Rev. Lett. 95, 177403 (2005).

[70] B.D. Gerardot, D. Brunner, P.A. Dalgarno, P. Öhberg, S. Seidl, M. Kroner, K. Karrai, N.G. Stoltz, P.M. Petroff, R.J. Warburton, Nature 451, 441 (2008).

[71] P. Hawrylak, Phys. Rev. B 60, 5597 (1999).

[72] K. Hinzer, P. Hawrylak, M. Korkusinski, S. Fafard, M. Bayer, O. Stern, A. Gorbunov, A. Forchel, Phys. Rev. B 63, 075314 (2001).

[73] A.S. Bracker, E.A. Stinaff, D. Gammon, M.E. Ware, J.G. Tischler, D. Park, D. Gershoni, A.V. Filinov, M. Bonitz, F. Peeters, C. Riva, Phys. Rev. B 72, 035332 (2005).

[74] J.I. Climente, A. Bertoni, G. Goldoni, Phys. Rev. B 78, 155316 (2008).

[75] R. Loudon, The Quantum Theory of Light, Claredon Press, Oxford 1973.

[76] R.S. Knox, Theory of Excitons, Academic Press, New York 1963.

[77] A. Zrenner, L.V. Butov, M. Hagn, G. Abstreiter, G. Böhm, G. Weimann, Phys. Rev. Lett. 72, 3382 (1994).
[78] M. Feucker, R. Seguin, S. Rodt, A. Hoffman, D. Bimberg, Appl. Phys. Lett. 92, 63116 (2008).

[79] T.H. Stievater, X. Li, D.G. Steel, D. Gammon, D.S. Katzer, D. Park, Phys. Rev. B 65, 205319 (2002).

[80] T. Smolenski, T. Kazimierczuk, M. Goryca, P. Kossacki, J. Gaj, P. Wojnar, K. Fronc, M. Korkusinski, P. Hawrylak, Acta Phys. Pol. A 119, 615 (2011).

[81] M. Baier, F. Findeis, A. Zrenner, M. Bichler, G. Abstreiter, Phys. Rev. B 64, 195326 (2001).

[82] Ł. Kłopotowski, M. Goryca, T. Kazimierczuk, P. Kossacki, P. Wojnar, G. Karczewski, T. Wojtowicz, Appl. Phys. Lett. 96, 201905 (2010).

[83] H.J. Krenner, M. Sabathil, E.C. Clark, A. Kress, D. Schuh, M. Bichler, G. Abstreiter, J.J. Finley, Phys. Rev. Lett. 94, 057402 (2005).

[84] M. Scheibner, T. Schmidt, L. Worschech, A. Forchel, G. Bacher, T. Passow, D. Hommel, Nature Phys. 3, 106 (2007). 